\title{
Intramedullary Diffuse Astrocytoma of The Spinal Cord in a 3-Year-Old Patient
}

\author{
Sergio Calero-Martinez*, Simeon Georgiev, Panagiotis Fistouris and Uta Schick \\ Department of Neurosurgery, Clemens Hospital, Academic Hospital of Munster University Hospital (UKM), Germany \\ *Corresponding author: Sergio Calero-Martinez, Department of Neurosurgery, Clemens Hospital, Academic Hospital of Munster University Hospital \\ (UKM), Munster, Germany
}

Submission: July 25, 2018; Published: August 17, 2018

\begin{abstract}
We present the case of a 3-year old female with a diffuse astrocytoma (WHO grade II, IDH Wild Type) of the thoracic cord. The patient is without tumor-progression after 1-year follow-up. Guidelines at this age group are missing. After reviewing the literature, surgical tumor removal is recommended, however chemo- or radiotherapy or combined after surgical removal is controversial. This decision seems to be simplified by tumor grade and progression, as well as the age of the patient resulting in better outcomes. Radiotherapy is not performed in children below 5 years old. Early neurological rehabilitation is mandatory.
\end{abstract}

Keywords: Diffuse astrocytoma; Spinal cord tumor; Childhood

Abbreviations: WHO: World Health Organization; IDH: Isocitratede Hydrogenase; NF1: Neurofibromatosis Type 1; MRI: Magnetic Resonance Imaging; SEP or MEP: Somatosensory- and Motor-Evoked Potential Monitoring; CUSA: Cavitronic Ultrasonic Surgical Aspirator

\section{Introduction}

Intramedullary spinal cord tumors are rare neoplasms in the childhood and can potentially lead to severe neurological deterioration, decreased function, poor quality of life, or death. The most common lesions within these tumors are ependymomas, astrocytomas, and hemangioblastomas [1]. Astrocytomas are generally infiltrative in nature and characterized by a poorly defined plane. They represent around $30 \%$ of all spinal cord tumors in adults, while $90 \%$ in pediatric patients. In the pediatric population are mostly low-grade tumors [2]. Circa 20\% of these lesions are associated with [3]. We present a case of a child with a giant astrocytoma in the thoracic spinal cord. In our experience a total resection of the tumor under intraoperative neuromonitoring should be attempted with early neurological rehabilitation to achieve better post term outcomes.

\section{Case Report}

A 3-year-old patient was admitted in Pediatric Neurosurgery in Clemenshospital Munster with complaints of chronic abdominal pain, obstipation and progressive gait disorder leading to frequent falls. The symptoms started almost one and half year ago, before the patient was able to walk, her medical history showed a multicystic dysplastic left kidney. The clinical and neurological examination reveals lumbar hypelordosis, radicular pain in both legs, pronounced ataxia and decreased muscle strength of lower extremities (Grade 3/5).

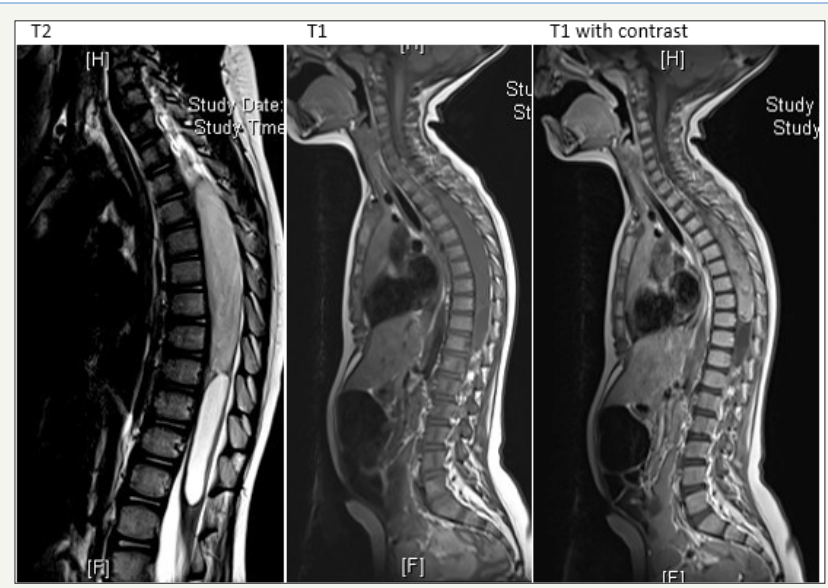

Figure 1: MRI images showing an intramedullary tumor from T3-T9 with enhancement in T2-weight and contrast enhancement and accompanied with a syrinx. 
Brain and whole spine magnetic resonance (MRI) were performed. Cranial MRI was normal, however the thoracic spine MRI with contrast administration revealed a $2 \times 2 \times 14 \mathrm{~cm}$ cystic mass with peripheral enhancement located at the level of T3-T9 vertebrae
(Figure 1) The lesion caused an expansion of the cord. T2-weighted images showed hyperintensity consistent with vasogenic edema, the differential diagnose favored a diffuse astrocytoma grade II.

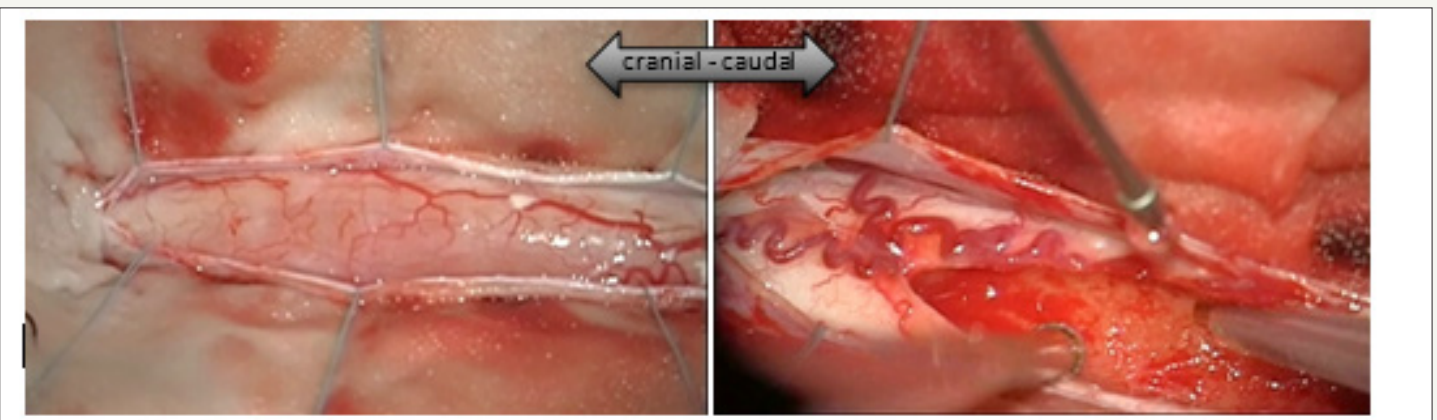

Figure 2: Intraoperative pictures showing the in-situ tumor and its resection starting at its poles.

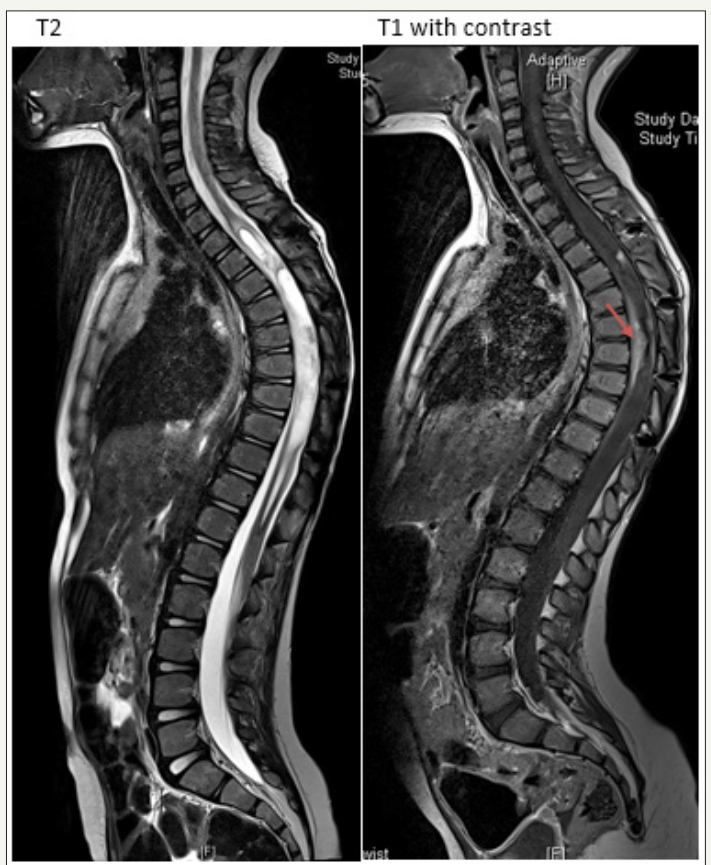

Figure 3: MRI images after 1-year follow-up. T1 with (a) and without (b) contrast showing no tumorprogression. The arrow indicates the residual tumor.

After reviewing the therapeutic options with the parents, neuropediatric and pediatric neuro oncology, surgical excision was decided. There are not guidelines for chemotherapy at this age. Steroids were applied before surgery. A multilevel osteoplastic laminoplasty from $\mathrm{T} 2 / 3$ to $\mathrm{T} 9 / 10$ was performed, particularly because of the extensive compression of the spinal cord. A midline myelotomy allowed us to reach the tumor immediately. Initial biopsy was obtained, the intra-surgical frozen section confirmed the diffuse astrocytoma. Cephalo-caudal tumor debulking with the CUSA was performed (Figure $2 \& 3$ ), because of the tumor infiltrations in the neuronal tissue only a subtotal resection was possible. The syrinx was also opened at both ends. During the procedure, the somatosensory evoked potentials (SEPs) showed a gradual delayed response from the left side, however no abnormal findings were seen in the MEP monitoring and the neurological status of the patient was unchanged after surgery. The histology showed a diffuse fibrillary Astrocytoma WHO-Grade II. Postoperatively, the patient exhibited some preexisting mild bladder disturbances further on and slight kyphosis requiring physiotherapy. The patient was taken into our neuropediatric rehabilitation, after one month she showed better outcomes in areas of functionality, disposition and interaction and could be released. No further adjuvant therapies were implemented.

After one year, the MRI images show only the residual tumor without progression. The patient did not show any further clinical alterations, instead she exhibited better neurological status (McCormick grade II), mobility and muscular strength.

\section{Discussion}

Astrocytomas are the most frequent pediatric intramedullary spinal cord tumors, however due to the rarity of occurrence, these 
lesions are often difficult to detect and are often misdiagnosed. The neurological deficits and clinical features include abdominal and/ or back pain, radicular pain, motor and/or sensory deficits, bladder or bowel alterations. The gold standard for diagnosis is an MRI with contrast administration. After diagnosis, optimal management with surgical tumor excision, chemotherapy and radiotherapy must be considered [4]. Due to the progressive neurological deterioration and extensive tumor growth, the decision for surgical debulking was made. Chemotherapy was felt to be unclear in response facing the threatening paraparesis. In order to minimize spine deformities after surgery, we decided to perform a multilevel osteoplastic laminotomy. Surgical approaches for spinal tumor resection under intraoperative neuromonitoring are nowadays the preferred treatment option. Adjuvant therapies have showed an ambiguous role in the literature $[5,6]$. Given subtotal tumor excision, tumor progression, tumor grade and clinical features, chemotherapy and radiotherapy are recommended after surgery [7]. Nonetheless, radiotherapy is not feasible in young children due to possible deformities and development disorders attributed to it. The role of ergo and physiotherapy has been well described after surgical procedures in pediatric patients. In our experience, inpatient rehabilitation programs are the best option for those patients, in spite of their increased co-morbidity from the disease process itself, acute oncological management and neurological sequelae [8].

\section{Conclusion}

Although spinal cord tumors are rare, they have an increased impact in morbidity and mortality. Astrocytomas represent the most common histological finding. A multi-disciplinary team is necessary to provide comprehensive assessment and treatment, including neuropediatric, neurooncology, neuropsychology, pediatric neurosurgery, neuro-electrophysiology and physiotherapy. Complete resection of the tumor should be intended, using techniques such as intraoperative monitoring and multilevel osteoplastic laminotomy to reduce neurological alterations and spine deformations after surgery. Adjuvant therapies must be considered in patients with subtotal resection or tumor recurrence/ progression. Early neurological rehabilitation is preferred.

\section{Ethical Statement}

Funding: No funding was provided for this research

Conflict of interest: The authors declare that they have no conflicts of interest.

Informed consent: The patient's kin has consented to submission of this case report to the journal.

\section{References}

1. Samartzis D, Gillis C, Shih P, O’Toole J, Fessler R (2015) Intramedullary spinal cord tumors: Part I: Epidemiology, pathophysiology, and diagnosis. Global Spine J 5: 425-435.

2. Houten J, Cooper P (2000) Spinal cord astrocytomas: presentation, management and outcome. J Neurooncol 47(3): 219-224.

3. Samii M, Klekamp J (1994) Surgical results of 100 intramedullary tumors in relation to accompanying syringomyelia. Neurosurgery 35(5): 865-873.

4. Benes V, Barsa P, Benes V Jr, Suchomel P (2009) Prognostic factors in intramedullary astrocytomas: a literature review. Eur Spine J 18(10): 1397-1422.

5. Ahmed R, Menezes AH, Awe 00, Torner JC (2014) Long-term disease and neurological outcomes in patients with pediatric intramedullary spinal cord tumors. J Neurosurg Pediatr 13(6): 600-612.

6. Bouffet E, Pierre-Kahn A, Marchal J, Jouvet A, Kalifa C, et al. (1998) Prognostic factors in pediatric spinal cord astrocytoma. Cancer 83(11): 2391-2399.

7. Guss Z, Moningi S, Jallo G, Cohen K, Wharam M, Terezakis S (2013) Management of spinal cord astrocytomas, outcomes with adjuvant radiation. Int J Radiat Oncol Biol Phys 85(5): 1307-1311.

8. Raj V, Lofton L (2013) Rehabilitation and treatment of spinal cord tumors. J Spinal Cord Med 36(1): 4-11.
Creative Commons Attribution 4.0 International License

For possible submissions Click Here

\section{Submit Article}

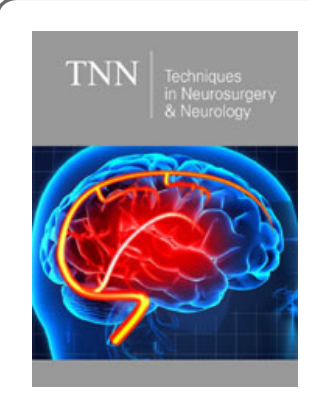

\section{Techniques in Neurosurgery \& Neurology}

\section{Benefits of Publishing with us}

- High-level peer review and editorial services

- Freely accessible online immediately upon publication

- Authors retain the copyright to their work

- Licensing it under a Creative Commons license

- Visibility through different online platforms 